\title{
Condition Code
}

National Cancer Institute

\section{Source}

National Cancer Institute. Condition Code. NCl Thesaurus. Code C93536.

A coded value specifying the state of being of an entity. 